\title{
Subjectivity in personal storytelling with visualization
}

Keywords: visualization, personal visualization, storytelling, personal narratives, subjectivity

In this article we explore visualization for personal storytelling and investigate techniques for communicating subjective experiences in personal visual narratives. Personal stories are often subjective and storytellers omit, make up, or embellish details to craft engaging stories or to communicate a perspective. As growing personal data collections allow individuals to leverage visualizations, we explore how personal visual narratives can express subjectivity. From an analysis of personal visualizations created by data enthusiasts, designers and artists, we collect techniques for deliberately expressing subjectivity during data collection, processing, visual encoding, and presentation. Our results prompt a discussion about the role and potential of subjectivity in personal visual storytelling.

\section{Introduction}

Personal narratives are a fundamental form of human communication and serve as means for strengthening interpersonal relationships, communicating a value system, and offering self-presentation (Langellier 1989). On a daily basis, people share accounts of mundane and memorable episodes from a personal perspective and sometimes include humor, irony, or embellishments (Langellier 1989; Labov 1967). This subjective interpretation is an integral part of personal narratives and distinguishes them from merely factual reports (Robinson 1981).

While the value and central role of subjectivity is widely acknowledged in art and design (e.g., Manovich 2002; Dorst 2006), in the visualization research community objectivity is a core value (Jorgenson 1995), and minimalist representations that reduce or eliminate the "lie factor" are widely considered the ideal (Tufte 1983).

The strength of visualization as a communicative medium is frequently discussed in research (Gershon 2011; Kosara 2013; Segel 2010) and data journalism (Cairo 2012). However, portraying "human subjectivity" and conveying experiences through visualizations is predominantly explored in data art (Manovich 2002; Viégas 2007) and only hinted at by a few personal visualization researchers (Donath 2010; Thudt 2016). We aim to explore subjective expressions in more depth in the context of personal storytelling with visualization. This work exists at the intersection of art, design, and visualization research.

Personal narrative visualizations are visual data representations that tell stories about personal experiences from the point of view of the narrator. Today more and more people are using visualizations of their 
personal data collections to tell compelling stories about themselves, their lives, and their experiences.

In this article we investigate the emerging phenomenon of personal narrative visualizations and the expression of subjectivity in these visual stories. Based on an analysis of personal narrative visualizations, we describe and categorize techniques for subjective personal storytelling with visualizations. We collected personal visual narratives created by data enthusiasts as well as by professional designers and artists. From these narratives, we sought to identify ways in which authors incorporate subjective perspectives with presentations of data. By considering work from a variety of diverse contexts, we offer an integrative perspective of subjective expression in personal visual narratives. We found that subjective perspectives can be introduced at every step of visualization creation: during data collection and processing, visual encoding, and when refining the presentation. Our categorization sheds light on the diversity of subjective expressions in personal visual narrative practice and can help inform the design of new visualization tools for personal storytelling.

\section{Background and related work}

Subjectivity arises because individuals experience the world in different ways. Those experiences are characterized not only by objective facts, but also by personal feelings and attitudes. To provide the background for our exploration, we describe research on the role of subjectivity in personal narratives and then examine how subjectivity has been discussed in visualization research, information design, and data art.

\subsection{Subjectivity in personal narratives}

Personal narratives have been studied in a variety of disciplines including psychology, sociology, literary folklore, and history (see Langellier 1989 for an overview). The "homo narrans" theory considers personal narratives as an elementary human need and the most common form of discourse in everyday life (Linde 1993b). Kristin Langellier summarizes William Labov's (Labov 1967) definition of verbal personal narratives as accounts of personal experiences "from the point of view of a narrator who interprets the significance of the experience" (Langellier 1989: 245). This definition emphasizes the evaluative function of personal narratives, which reveals the narrator's subjective attitude through emphasis and personal interpretation (Labov 1967: 37), distinguishing personal narratives from reports (Robinson 1981).

Personal narratives are a means of self-evaluation (Linde 1993a), of sharing one's value system (Langellier 1989), of self-exploration, and of shaping identity (Labov 1967; Worthham 2001). Personal narratives can also evoke sympathy, approval, or amusement (Goffman 1974), show others how the narrator wants to be perceived (Linde 1993a; Linde 1993b), and create intimacy, trust, and social connections (Linde 1993b). For these important functions of personal narratives, a subjective interpretation of the described experience is beneficial.

\subsection{Subjectivity in visualization and data art}

The positive aspects of subjectivity are rarely discussed in the visualization research community. The community largely agrees that an objective visual representation that produces repeatable insights is the ideal. Subjectivity is viewed with caution and should generally be avoided. However, researchers have argued that completely eliminating subjectivity is impossible (Jorgenson 1995; Valle 2015; Van Wijk 2005). For example, the choice of a visual encoding is subjective as it relies on personal experience and intent (Jorgenson 1995; Van Wijk 2005). Several research efforts have explored alternative strategies for creating more "truthful" visualizations. 
Approaches include integrating multiple perspectives of the same data (see Baldonado 2000), visualizing errors and uncertainties in the data itself (see Bonnau 2015), and supporting provenance by making change histories transparent (see Ragan 2016). In their discussion of critical visualization, Marian Dörk et al. discuss potential benefits of subjective visualizations such as empowering creators to have their voices heard (Dörk 2013). We discuss the value of subjective choices as a means to communicate personal perspectives, feelings, and experiences in narrative visualizations. Subjectivity as discussed in this paper is therefore distinct from the prevalent and widely condemned notion of lying with statistics (Huff 1954) and visualization (Tufte 1983), which refers to deliberate deceit and misrepresentation of the truth.

In data art, subjectivity and the focus on a "point of view" is considered a defining quality (Manovich 2002; Viégas 2007). Portraying personal subjectivity allows artists to evoke emotional and visceral responses (Gaviria 2008). Fernanda Viégas and Martin Wattenberg suggest that techniques that are often considered unconventional in visualization design, such as distortion or cluttered representations can be means to communicate artistic intent (Viégas 2007).

While storytelling is a common topic of discussion in the field of visualization (Cairo 2012; Gershon 2011; Kosara 2013; Segel 2010; Lee 2015), there is little research on personal storytelling with visualization. In their survey, Huang et al. identify sharing, privacy, and personalization as challenges for personal visualizations (Huang 2015), hinting at the importance of giving the data owners control over representations and the messages they convey. Donath et al. define the related concept of "data portraits" as "subjective renderings" for "self-representation" (Donath 2010), but do not describe specific techniques for creating such subjective representations. Similarly, Thudt et al. describe "expressing subjectivity" as a design goal for visual mementos and provide an example system as a proof of concept, but do not examine the variety of options available for creating subjective representations (Thudt 2016). Our investigation complements these discussions of the value of subjectivity in personal visualizations and provides an initial list of techniques for introducing deliberate subjectivity.

\section{Techniques for deliberate subjectivity}

To understand how subjective perspectives can be communicated in personal narrative visualizations, we examined a set of examples created in a personal context as well as work by professional visualization designers and artists. Some of these examples use data to provide accounts of personally significant or sentimental episodes that are similar to personal stories shared by individuals in everyday life. Other examples are artistic and more conceptually complex. We deliberately include a broad spectrum of examples to illustrate the diversity of techniques applied to express subjectivity in visual accounts of personal experiences. We collected examples from visualization blogs such as flowingdata.com and infosthetics.com using tags and keywords referring to personal visualizations (e.g., "personal", "selfsurveillance"), as well as from vis4me.com, which focuses on personal visualizations. We manually sampled a collection of over 100 initial results to identify a diverse set of 20 personal narrative visualizations that present data in a subjective fashion (indicated using [E1], [E2], etc.-see appendix). We excluded descriptions of lifelogging devices or applications and focused on stories about personal experiences from the point of view of the narrator. We also sampled to maximize the diversity of approaches and creators. However, we do not claim that our list of techniques is comprehensive. Figure 1 shows an overview of the subjectivity techniques identified from the collection we explored along with selected examples of each. 


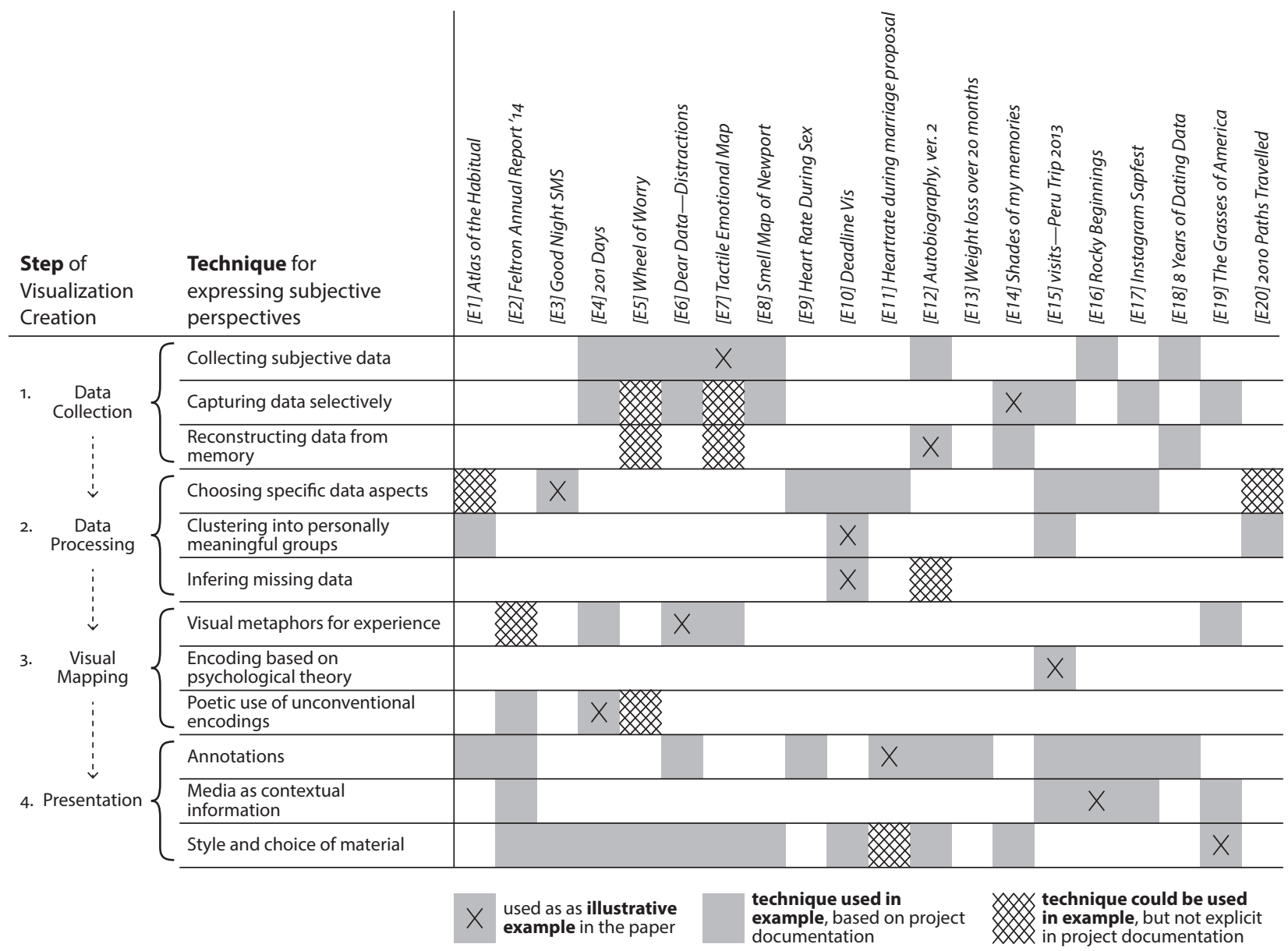

Figure 1. Overview of techniques for introducing subjectivity in personal narrative visualizations with associated examples.

Three researchers collaboratively analyzed the collection of examples using open coding. In the first coding pass a coarse categorization of techniques emerged, describing visualization creation steps during which subjectivity can be introduced: (1) data collection, (2) data processing, (3) visual encoding, and (4) presentation.
Steps 2 to 4 align with the widely used pipeline for creating information visualizations (Card 1999).

Our categorization extends the pipeline by including data collection (1), since it can be an integral part of the creation of narratives, particularly if narrators collect data specifically for this purpose. In a second pass 
we refined our categorization and identified several specific techniques that fit within each step (see Figure 1, column 2).

Given that documentation for each project was limited or not available, we relied on our own subjective interpretation of the work to define the use of a given technique. In Figure 1 we distinguish between explicit uses of subjectivity techniques described in the project documentation (descriptions and images) and potential uses of techniques that we identified during coding. Many examples use multiple techniques concurrently. In the following sections, we describe techniques for each step and illustrate each by discussing one example (highlighted with an $x$ in Figure 1).

\subsection{Data collection}

During data collection, narrators can introduce subjectivity by (1) collecting inherently subjective data, (2) capturing selectively, and (3) reconstructing data from memory.

3.1.1 Collecting subjective data. Some types of data, such as dreams, hopes, emotions etc., inherently include subjective perspectives. Such data originates from the point of view of the narrator and cannot be separated from its personal interpretation. We exclude the use of biosensors to infer emotional states from measurable body data (e.g., heart rate, sweat level, etc.), as such data does not contain the narrator's interpretation.

Using subjective data as a basis for visual representations dates back to the 50 and 6os with the Situationists' "psychogeography" (Pinder 1996). Members of this political art movement appropriated geographic maps to investigate "the actual human experience of a city" (Lynch 1984), for instance "the evident division of a city into zones of distinct psychic atmospheres" (Debord 1981: 6-7).
Kate McLean, a contemporary artist inspired by Situationist psychogeography, also uses subjective data in her sensory maps ([E7][E8]). Her "Emotional Tactile Map" ([E7], see Figure 2) shows the artist's emotional state during her MFA. The wooden visualization is inspired by Inuit coastal maps and contrasts fear and elation on one side and surprise and anticipation on the other.

\subsubsection{Capturing data selectively. Autobiographical} memories are a selective repository of experiences that are filtered by people's evaluation of their significance (Conway 2000). Selective data capture entails a manual collection process where each potential data point is individually assessed for its personal significance or relevance to an intended story. Unlike when capturing subjective data (see Section 3.1.1), here the data itself is not necessarily ambiguous. Instead, subjectivity is introduced through the active selection of certain data points and the omission of others. The practice of taking

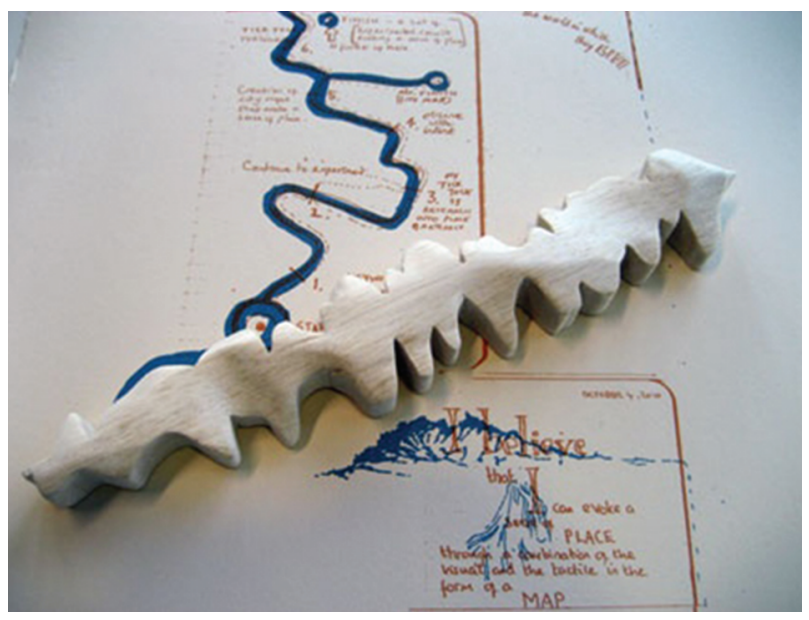

Figure 2. McLean's "Emotional Tactile Map" visualizes the artist's emotional state [E7]. 
personal photos is a familiar example of this technique.

By consciously deciding if and how a particular moment should be recorded-rather than using automatic logging devices running in the background-people create subjective data collections.

Alice Thudt used selectively captured photo collections as data source to show which colors she perceived as characteristic for Indian cities visited during a trip [E14]. She algorithmically extracted dominant colors from her photo collections in which she gives subjective preference to certain scenes over others. In a second iteration of the project Thudt created watercolor paintings inspired by the output of the algorithm and the city map outlines (see Figure 3). Before painting she selected and corrected the set of color values to bring them closer to her memory, hence adding an additional layer of subjectivity to the data source.

3.1.3 Reconstructing data from memory. When reconstructing data from memory, rather than capturing it in the moment, the collected data inevitably contains subjective elements. Autobiographical memory is an inherently subjective view on the past that is constantly reinterpreted and adjusted (Conway 200o). Therefore, data that is reconstructed from memory is a retrospective subjective interpretation of personal experiences.

We assume that Ward Shelley's 50-year autobiography ([E12], see Figure 4) was reconstructed from memory. It shows how different areas of his life evolved over time. Some aspects of the data, such as his philosophies and identities, show a strong subjective interpretation based on retroactive reflection.

\subsection{Data processing}

During data processing narrators clean, filter, and prepare data for visualization. Techniques for introducing subjectivity during data processing include (1) choosing specific aspects of the data to visualize, (2) clustering data to create personally meaningful groupings, and (3) inferring data points from other sources.

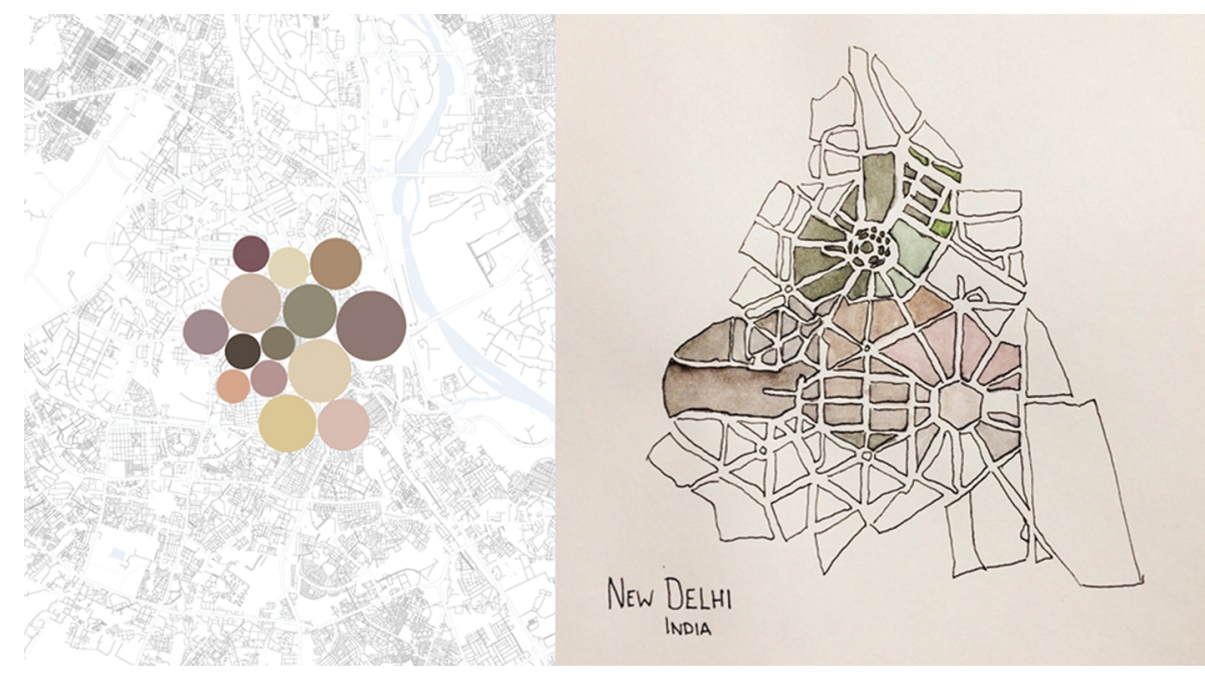

Figure 3. (a) Algorithmically extracted colors from selectively captured travel pictures; (b) an adaption of the algorithmic output painted with watercolors and adjusted to match personal memories of New Delhi [E14]. 

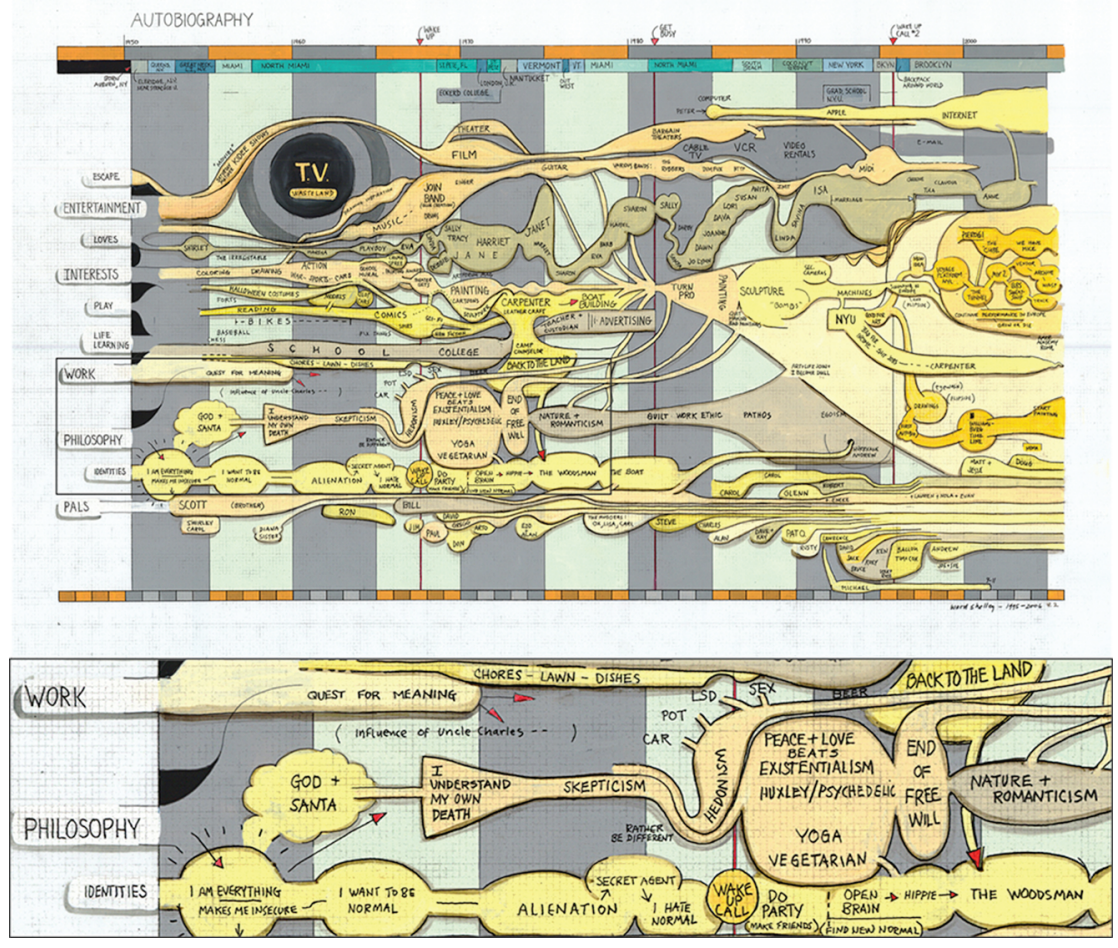

Figure 4. Shelley's visual autobiography uses data that appears to be reconstructed from memory [E12].
3.2.1 Choosing specific data aspects. Specific aspects of an existing data source can be extracted to support a point of view, to isolate personally meaningful excerpts, or to hide data that the narrator does not want to share. This can be achieved by filtering a dataset based on criteria that the narrator identifies as relevant for the intended story, or by manually processing the data and selecting which data to include or omit.

To create a necklace for his girlfriend, Paul Heinicker extracted all good night SMS from a 2-year long chat history [E3]. The necklace shows the frequency and length of the exchanged messages (see Figure 5). Rather than visualizing the entire communication record, Heinicker chose an aspect that contains personal meaning. In his description of the project, he discusses the process of isolating good night messages from the rest of the collection as a complex data-mining task.

3.2.2 Clustering data into personally meaningful groups. Clustering data points into groups is a common scientific technique that can be applied in personal visual narratives to convey a subjective interpretation of data. Personally meaningful clusters of values can be created algorithmically using subjectively chosen methods and parameters or constructed manually based on personally selected criteria. When individual logs are clustered, the grouping may contain additional personal meaning not inherent in the raw data and can therefore convey a subjective perspective. 


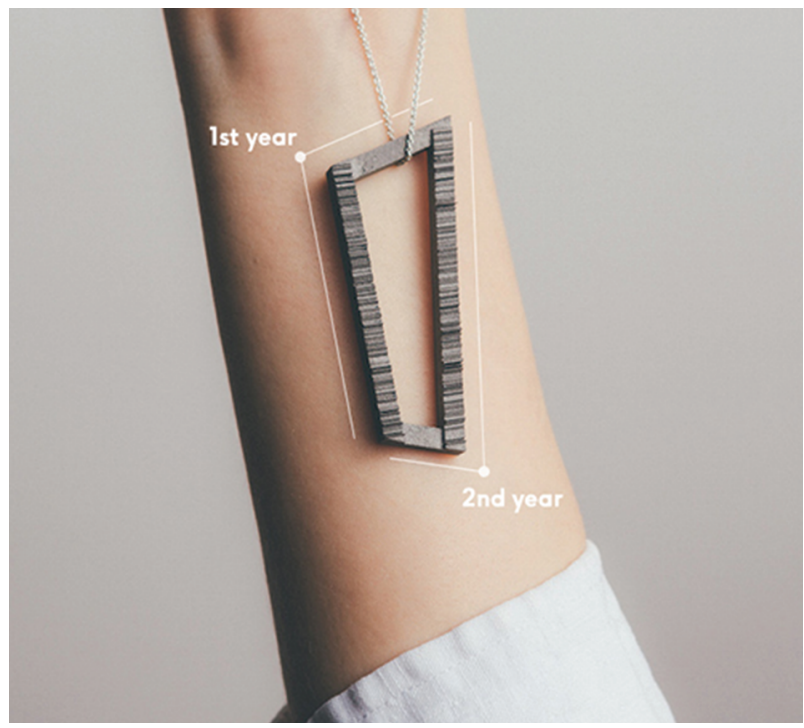

Figure 5. For Heinicker's necklace showing good night SMS, the relevant data is isolated through intricate data mining [E3].

In Charles Perin's visualization of his working habits and paper revisions during a conference deadline ([E10], see Figure 6), the clustering conveys personal meaning. Perin used Dropbox and Google Drive syncs as data sources to show paper revisions. However, these services create syncs for each minor edit, obscuring his intended notion of revisions. He therefore experimented with different thresholds to cluster syncs by the same author within a specific timeframe. The resulting clusters reflect his subjective sense of how the revisions for each paper progressed.

3.2.3 Inferring missing data. When personally relevant aspects of an experience were not recorded, narrators either reconstruct the missing data purely from memory (see Section 3.1.3) or infer it from existing data sources.
This requires choosing an appropriate data source as a basis and extrapolating the required data. Methods can be based on general heuristics (e.g., inferring the mode of transportation from the speed of movement) or include additional subjective interpretations based on narrators' personal experience and knowledge of their own habits.

Perin considered his lack of sleep nearing a deadline as an important element of his intended narrative ([E10], see Figure 6), but lacked sleep tracker data. He therefore inferred his sleep patterns from pedometer logs, counting six consecutive hours with no logged activities as sleep. The inferred sleep data is tied to his subjective sense of the relationship between sleep and steps during this time. His choice to include sleep patterns communicates a personal message about his intense work schedule.

\subsection{Visual encoding}

During visual encoding, narrators associate data with visual properties and create visual forms. While researchers have discussed the inevitable subjectivity in the choice of visual encoding (see Section 2.2), here we describe techniques for conveying a personal experience or message through the encoding: (1) visual metaphors for experiences, (2) layout algorithms based on psychological theory, and (3) unconventional encodings to create poetic representations.

3.3.1 Visual metaphors for experiences. Visual metaphors in visualizations suggest an association with an internal mental image and therefore shape the interpretation of the presented information (Ziemkiewicz \& Kosara 2008). They are widely used in narrative visualizations and can support memorability and interpretation of a visualization (Eppler 2006). By purposefully evoking a mental image that relates to a personal experience, narrators can express a point of view and make their experience more relatable. 


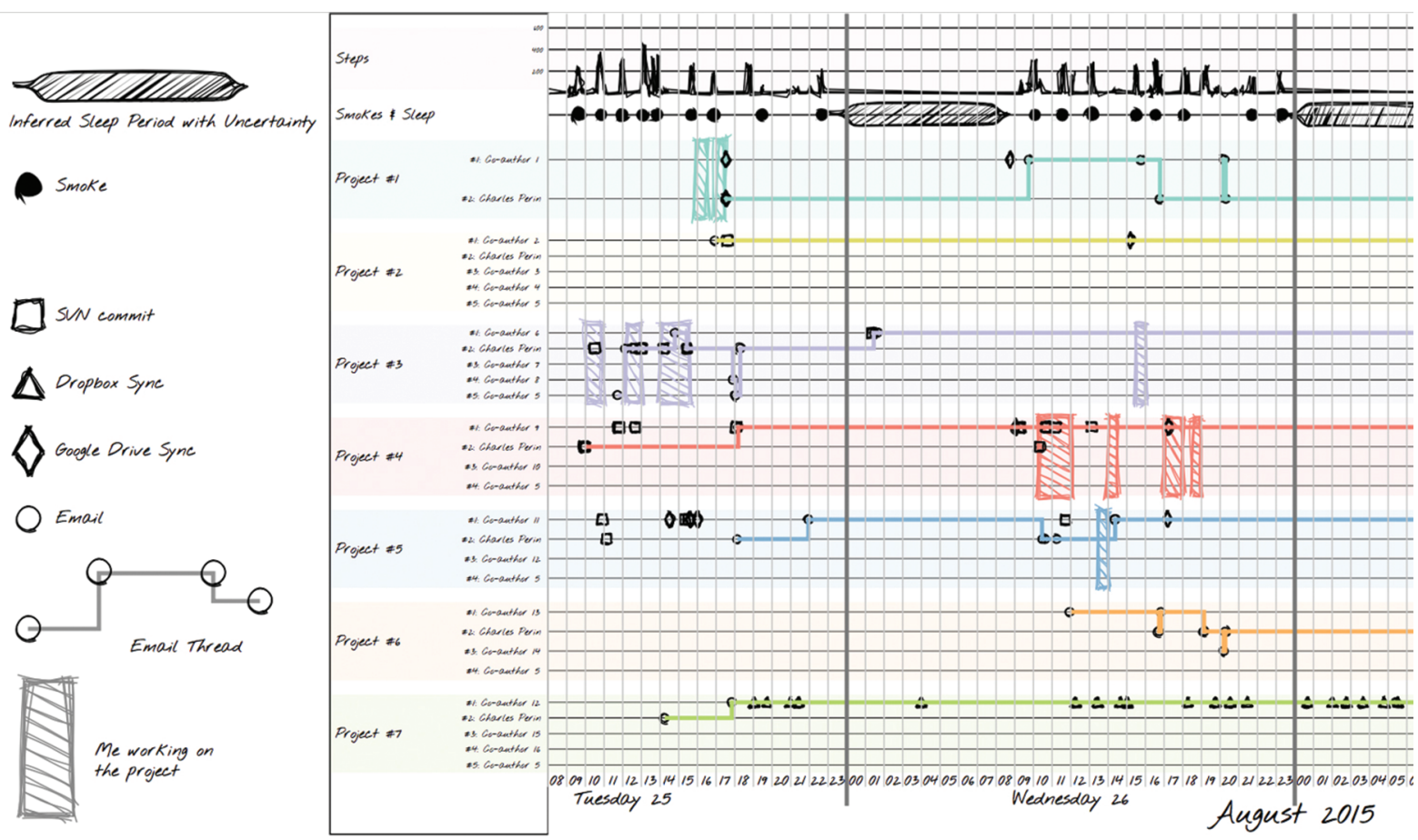

Figure 6. In this visualization of work during a paper deadline, the intended notion of paper revisions is conveyed by clustering Dropbox and Google Drive syncs into personally meaningful groups [E10]

In their "Dear Data" project, Giorgia Lupi and Stefanie Posavec sent hand-drawn postcards about one aspect of their lives to each other, each week over the course of one year [E6]. Figure 7 shows their distinct visual metaphors for "distractions". While Lupi showed them as drops symbolizing interruptions that dribble in unexpectedly, Posavec chose lines that "were drawn as though they were veering off-track, alluding to how I feel whenever I am distracted". Their distinct visual metaphors demonstrate how the same phenomenon can be experienced and encoded in personal ways that make their subjective experiences relatable.
3.3.2 Encodings based on psychological theory. Psychological theories model human thoughts, emotions, behaviors, and experiences based on empirical evidence. Such theories can be used for developing visual encodings that more closely reflect specific human experiences. This technique can be used to emphasize aspects to which people subjectively give more importance, or to align concepts that people experience as related. Narrators can further manually adjust such encodings to make them fit better with their subjective experiences.

When creating the tool "visits", Thudt et al. aimed to algorithmically encode location histories in a way that 

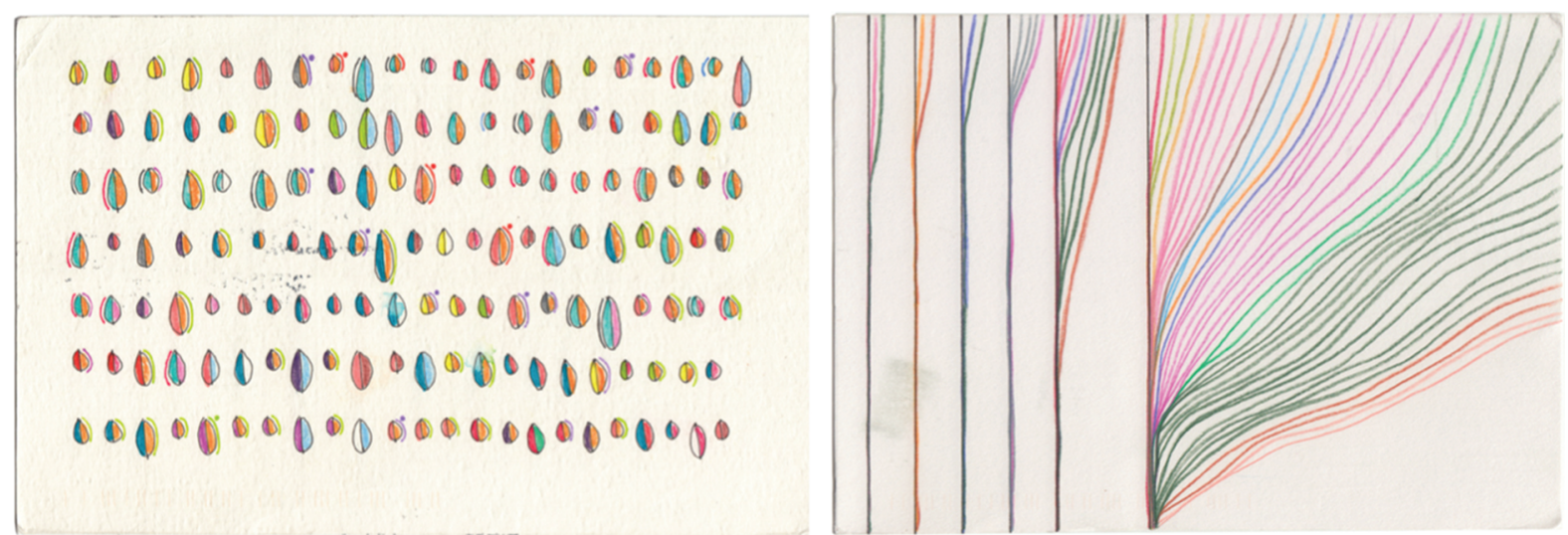

Figure 7. Dear Data showcases how two designers pick different visual metaphors to represent personal experiences (here, "distractions") [E6].

reflects how people remember their trips (Thudt 2016). Psychological theories suggest that episodes of autobiographical memories are organized both chronologically and spatially (Tulving 1972), and that people remember journeys as a sequence of visited places (Brown 1998). The map-timeline visualization in "visits" builds on these theories by encoding the data as a sequence of stays in different places. Figure 8 shows Thudt's trip to Peru in 2013 [E15]. She manually adjusted the timeframe to show this personally significant time period and chose to focus on stays at different geographic regions. Compared to common pins-on-a-map approaches, "visits" algorithmically creates an approximation of how the travel was experienced, by giving more emphasis to places where she spent more time. By customizing the time period of interest and by choosing to group points by region, Thudt created a representation that matches her subjective memory of the trip.

\subsubsection{Poetic use of unconventional encodings. Using} visual encodings in surprising or unconventional ways can add power to a personal message. For instance, Viégas and Wattenberg discussed how clutter or distortion can be used to expressively communicate an experience (Viégas 2007). While such encodings might distort or obscure the data, they can make emotional experiences relatable on a visceral level. Another way to create an element of surprise and stimulate reflection is the use of encodings in personal narratives that are typically applied in other contexts (e.g., business reports or scientific publications, see [E2]). Such an intentional breach between the type of data and its visual representation can reinforce a subjective message.

In her work, artist Katie Lewis visualizes data from different personal data sources, such as bodily sensations, in intricate webs made of physical pins and thread [E4]. The deliberately cluttered representations convey the chaotic and complex nature of human experience (see Figure 9). The deterioration of visual clarity alludes to the insufficiency of rigid systems and "scientific" encodings for capturing human sensations. 


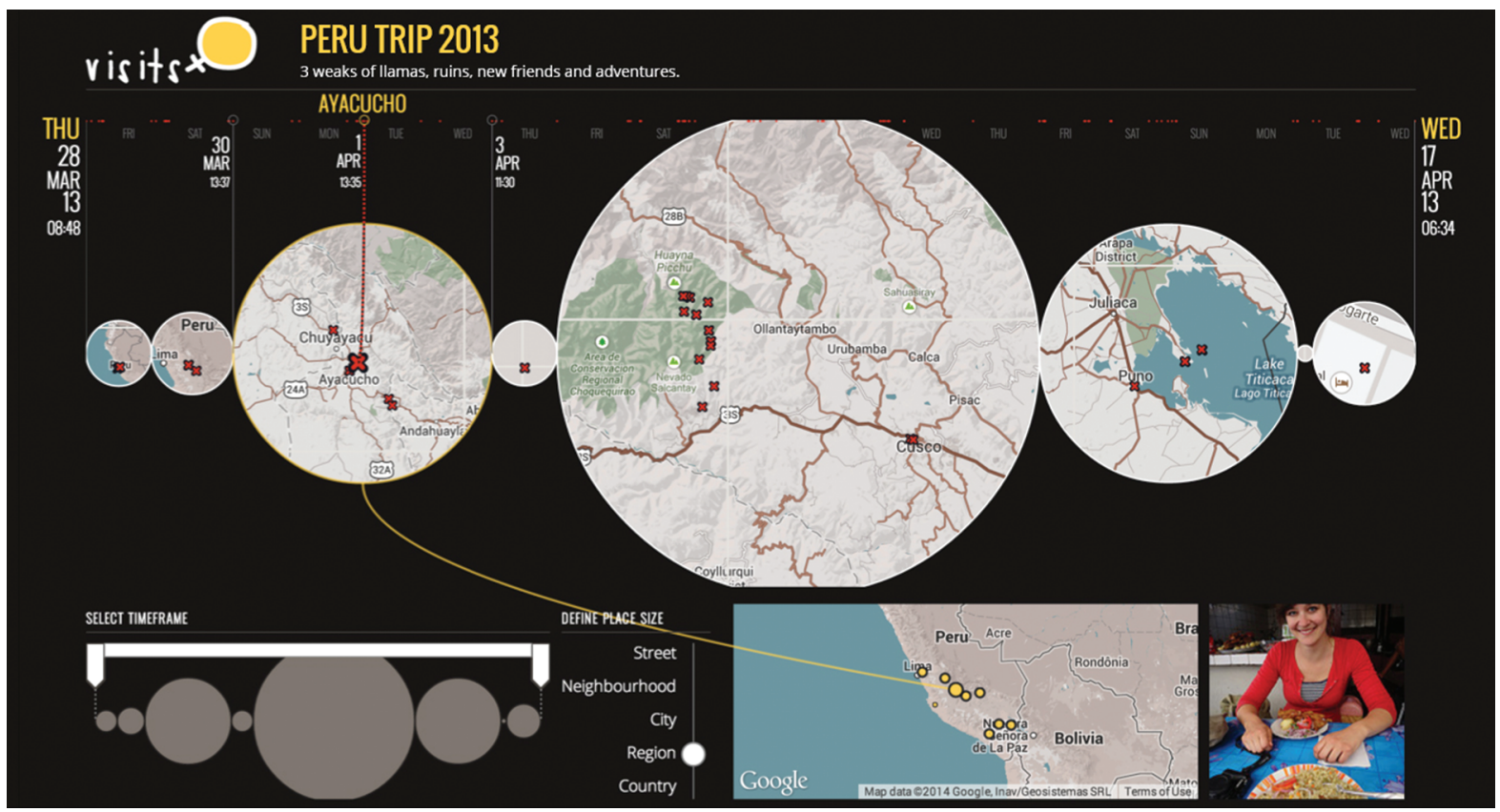

Figure 8. The tool "visits" uses an algorithmic encoding based on psychological theory to represent travel histories as a sequence of stays at different places [E15].
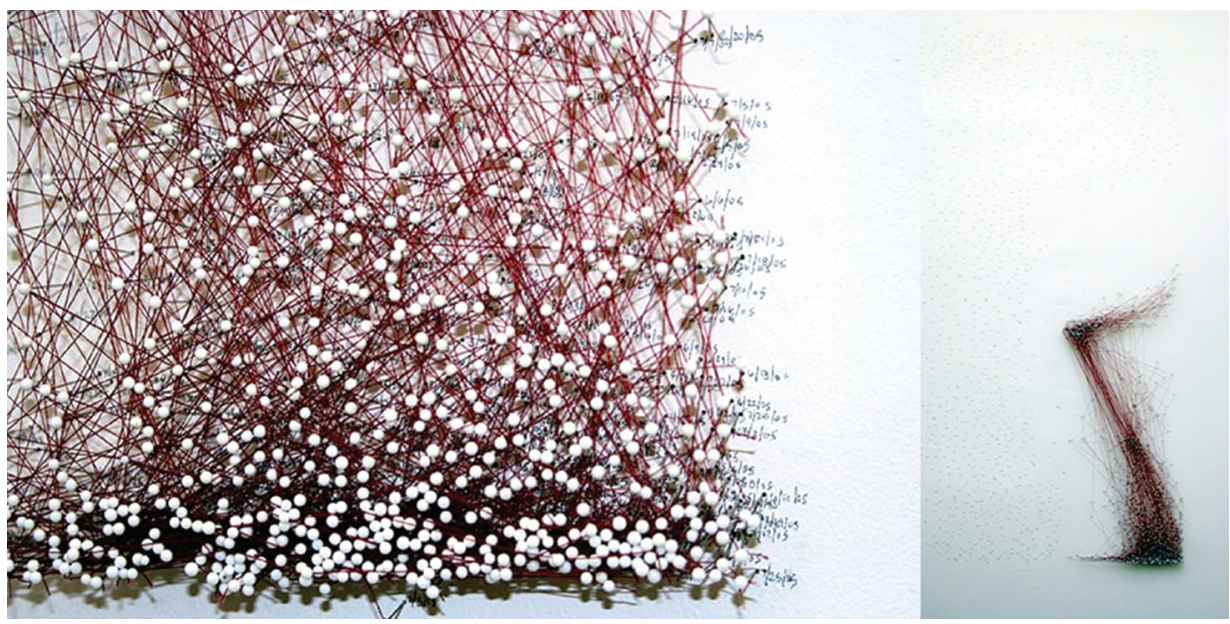

Figure 9. 201 Days shows the artist's sensations felt in different body parts. The clutter symbolizes the complexity of human experiences [E4]. 


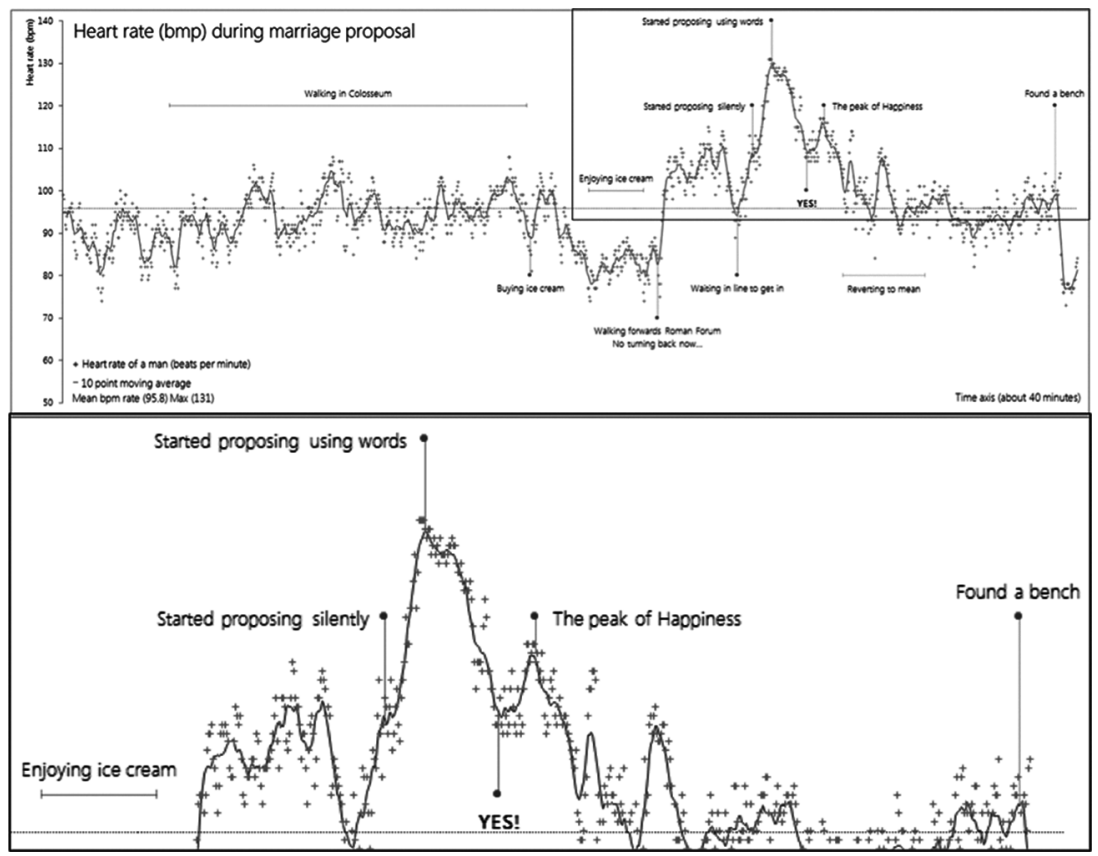

Figure 10. Visualization of the narrator's heart rate during his marriage proposal with humorous annotations [E11].
Although this example presents an incident rather than an episode from the life of the artist, it is included due to its focus on personal data.

\subsection{Presentation}

Narrative structure is mostly introduced during presentation, where the graphic is laid out and rendered. In this step, we observed (1) annotations, (2) the addition of media to provide context, and (3) personal choices of style and materials as ways to add subjective interpretations. While most techniques discussed earlier in this paper require a substantial level of expertise, adding a subjective interpretation through annotations or style is accessible to non-experts and often supported by tools for broader audiences.
3.4.1 Annotations. Annotations are a common way to introduce narrative in visualizations (Segel 2010). In personal visual narratives, annotations can convey personal interpretations that turn a visual report into a personal story. Annotations can add interpretations to the graph as a whole, or point out personally significant points in the visualization.

We found several examples of annotated personal stories created with widely accessible tools such as Microsoft Excel or the Fitbit App ([E9][E11][E13]). One example shared on Imgur shows a simple line graph of the heart rate during the narrator's marriage proposal that is annotated with brief anecdotes ([E11], see Figure 10), while another depicts weight gains and losses by pointing out the causes, such as "all-inclusive holidays", "new year's resolution", and a breakup [E13]. The simple 
annotations turn these visualizations into personal and humorous narratives.

\subsubsection{Media as contextual information. Inclusion of} media (e.g., photos, audio, hand-drawn sketches) in visual narratives can add personal context and enrich representations with glimpses of individual experiences. Photos are familiar and accessible ways to capture personal experiences. Similarly, music can be powerful in expressing emotions. Some services for sharing personal workout data (e.g., Endomondo or Garmin connect) incorporate features for enhancing visual stories with photos. We found storytelling examples that integrate a broad variety of media as contextual information, including photos ([E2][E15][E17], see e.g., Figure 8), hand-drawn sketches ([E7], see e.g., Figure 2), flipbooks that create an animation from automatically captured photos ([E19], see e.g., Figure 11), and music ([E16]).

In "Rocky Beginnings", for instance, the narrator enhances the visualization of her music listening history and associated emotions with short audio snippets [E16]. This additional contextual information allows the audience to relate to the narrator's subjective experience of moving to a new city and provides an emotional musical background for the visualization.

3.4.3 Style and choice of material. In most of the examples we analyzed, narrators adjusted the style of the representation, including fonts, color schemes, or physical materials to reflect their own stylistic preference. Some examples, however, convey a deeper symbolic meaning through stylistic choices that go beyond surface aesthetics. For instance, Perin's representation uses sketchy rendering to convey uncertainty in his data collection and processing ([E10], see Figure 6).

In physical personal visualizations the choice of material becomes an important way to express a subjective perspective beyond stylistic preferences. For example, Julia Lines Wilson weaves tapestries from grasses found on walks in chronological order to visualize her movement ([E19], see Figure 11). By using these materials, she allows the audience to relive her personal experience of her surroundings.

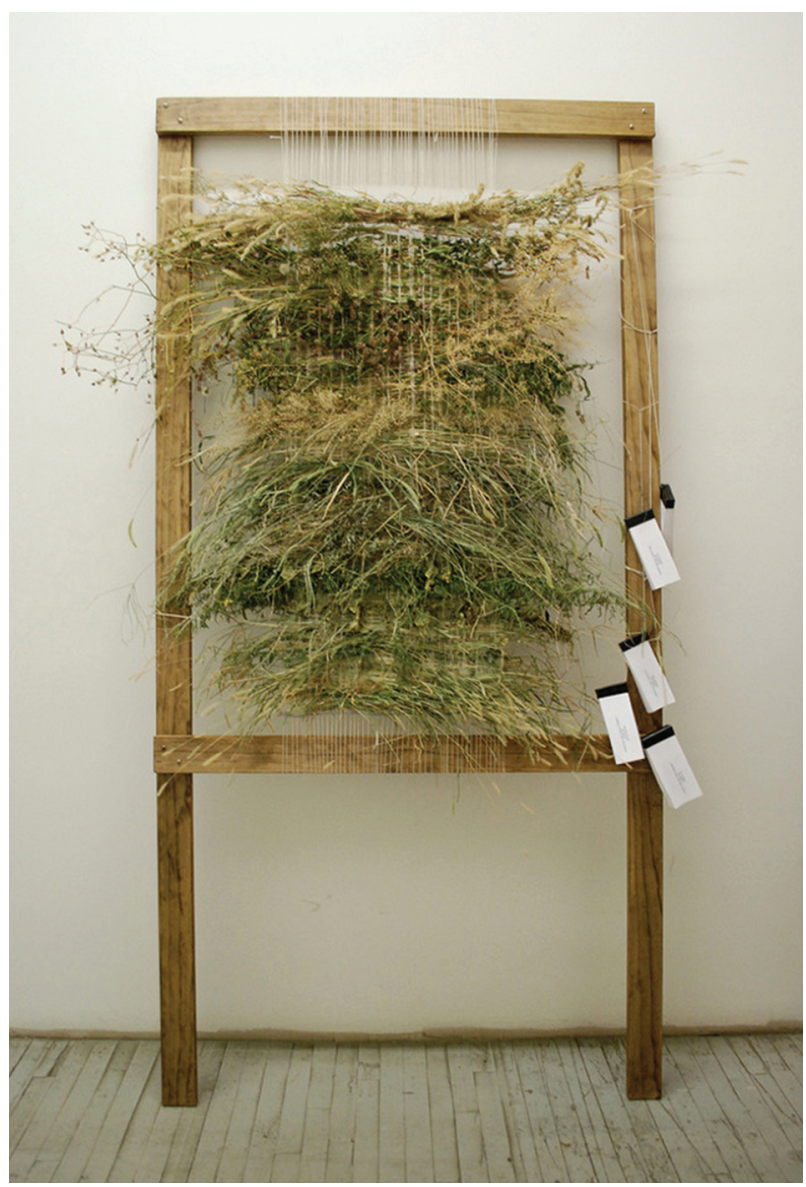

Figure 11. Wilson's physical visualization incorporates grass found on a walk as material, allowing the audience to relate to her experience of her surroundings [E19]. 


\section{Discussion}

While research in visualization predominantly recommends objective data representations, we think that a discussion of personal and subjective approaches, such as those discussed in the art and design communities, can benefit the creation of narrative visualizations. Through our initial categorization of techniques for subjective visual storytelling we intend to open a broader discussion of subjectivity in personal visualization and outline opportunities for future research.

We presented several benefits of subjective displays of personal data. However, as in all media, it can sometimes be difficult to draw a clear boundary between a subjective account and a misrepresentation. In verbal communication people have learned reading between the lines and interpreting certain forms of rhetoric, such as exaggerations. When we use visualizations to tell personal stories, it is probable that a high degree of visual literacy is required to understand similar subtleties. Furthermore, we need to better understand how to make subjective interpretations of an experience apparent. Identifying techniques that indicate the extent to which a representation conveys a personal perspective is an exciting avenue for future research that can have impact beyond personal visual storytelling.

The expression of the narrator's sense of humor and irony is part of the appeal of personal narratives. So far, it is not well understood how such subtle undertones can be conveyed in visualizations. In the examples we presented, we identified some particularly amusing narratives. Humor can originate from data collection choices, as in Shelley's autobiography (see Figure 4), or from particularly amusing annotations, as in the visualization of the heart rate during a marriage proposal (see Figure 10). Investigating techniques for conveying humor and wit through visualizations could be a valuable addition to the ongoing discussion of storytelling with visualization.
Most subjectivity techniques, especially during data collection, processing and visual encoding, require considerable skills and strong data and visualization literacy. Didactic approaches that teach people necessary skills could empower wider audiences to tell their own stories with data. However, further research and development is necessary to provide accessible and expressive personal visualization tools that allow narrators to do more than just annotate and restyle existing charts. These could include systems that let narrators edit, remove, add, and cluster data on the fly in order to visually curate narratives. Physical and digital tools that let novices manipulate or even invent new visual encodings could also make it easier for novice narrators to express their personal perspectives.

We explored the role of subjectivity for creating compelling personal narratives with visualization by illustrating how people are injecting personal meaning in all steps of the creative process. Our initial categorization sheds light on the various ways in which everyday narrators as well as professional designers and artists use visualization as a medium to tell subjective personal stories. This work can further inform the development of visualization tools for personal storytelling, making the creation of subjective personal narratives accessible to even wider audiences.

Submission date: 10 October, 2016

Accepted date: 8 February, 2017

\section{References}

Baldonado, M.Q.W., Woodruff, A. \& Kuchinsky, A. (2000). Guidelines for using multiple views in information visualization. In Proceedings of AVI (pp. 110-119). ACM.

Bonneau, G.P., Hege, H.C., Johnson, C.R., Oliveira, M.M., Potter, K., Rheingans, P. \& Schultz, T. (2014). Overview and state-ofthe-art of uncertainty visualization. In Scientific Visualization (pp. 3-27). Springer, London. 
Brown, N.R. \& Schopflocher, D. (1998). Event cueing, event clusters, and the temporal distribution of autobiographical memories. Applied Cognitive Psychology, 12(4), 305-319. doi: 10.1002/ (SICI)1099-0720(199808)12:4<305::AID-ACP569>3.0.CO;2-5

Cairo, A. (2012). The Functional Art: An introduction to information graphics and visualization. New Riders.

Card, S.K., Mackinlay, J.D. \& Shneiderman, B. (1999). Readings in information visualization: using vision to think. Morgan Kaufmann.

Conway, M.A. \& Pleydell-Pearce, C.W. (2000). The construction of autobiographical memories in the self-memory system. Psychological review, 107(2), 261-288. doi: 10.1037/0033-295X.107.2.261

Debord, G. (1981). Introduction to a critique of urban geography. Situationist International Anthology, 5-8.

Donath, J., Dragulescu, A., Zinman, A., Viégas, F. \& Xiong, R. (2010). Data portraits. Leonardo, 43, 375-383. doi: 10.1162/LEON_a_00011

Dörk, M., Feng, P., Collins, C. \& Carpendale, S. (2013). Critical InfoVis: Exploring the politics of visualization. $\mathrm{CHI} 13$ Extended Abstracts on Human Factors in Computing Systems, 2189-2198. doi: 10.1145/2468356.2468739

Dorst, K. (2006). Design problems and design paradoxes. In Design issues, 22(3), 4-17. DOI: 10.1162/desi.2006.22.3.4

Eppler, M.J. (2006). A comparison between concept maps, mind maps, conceptual diagrams, and visual metaphors as complementary tools for knowledge construction and sharing. Information visualization, 5(3), 202-210. doi: 10.1057/palgrave.ivs.9500131

Gaviria, A.R. (2008). When is information visualization art? Determining the critical criteria. Leonardo, 41(5), 479-482. doi: 10.1162/leon.2008.41.5.479

Gershon, N. \& Page, W. (2001). What storytelling can do for information visualization. Communications of the ACM, 44(8), 31-37. doi: 10.1145/381641.381653

Goffman, E. (1974). Frame analysis: An essay on the organization of experience. Harvard University Press.

Huang, D., Tory, M., Aseniero, B.A., Bartram, L., Bateman, S., Carpendale, S., Tang, T. and Woodbury, R. (2015). Personal visualization and personal visual analytics. IEEE TVCG. 21 (3): 420-433.

Huff, D. (1954). How to lie with statistics. NY: Norton.
Jorgenson, L., Kritz, R., Mones-Hattal, B., Rogowitz, B. \& Fraccia, F.D. (1995). Panel: Is visualization struggling under the nyth of objectivity?. Proceedings of IEEE Visualization. 412-415.

Kosara, R. \& MacKinlay, J. (2013). Storytelling: The next step for visualization. Computer, 46(5): 44-50. doi: 10.1109/MC.2013.36

Labov, W. \& Waletsky, J. (1967). Narrative analysis: Oral versions of personal experience. Essays in the Verbal and Visual Arts. Ed. J. Helms, 12-44.

Langellier, K.M. (1989). Personal narratives: Perspectives on theory and research. Text and Performance Quarterly, 9(4): 243-276. doi: 10.1080/10462938909365938

Lee, B., Henry Riche, N., Isenberg, P. \& Carpendale, S. (2015) More than Telling a Story: A Closer Look at the Process of Transforming Data into Visually Shared Stories. IEEE CG\&A, 35(5): 84-90.

Linde, Charlotte (1993). Narrative and the iconicity of the self. Life Stories: The Creation of Coherence. New York: Oxford University Press. 98-126.

Linde, Charlotte (1993). What is a life story? Life Stories: The Creation of Coherence. New York: Oxford University Press. 20-50.

Lynch, K. (1984) Reconsidering the image of the city. Cities of the Mind: Images and Themes of the City in the Social Sciences. 151-161.

Manovich, L. (2002). The anti-sublime ideal in data art. Retrieved from http://meetopia.net/virus/pdf-ps_db/ LManovich_data_art.pdf

Pinder, D. (1996). Subverting cartography: The Situationists and maps of the city. Environment and Planning A, 28(3), 405-427. doi: 10.1068/a280405

Thudt, A., Baur, D., Huron, S. \& Carpendale, S. (2016). Visual Mementos: Reflecting memories with personal Data. IEEE TVCG, 22(1), 369-378.

Ragan, E.D., Endert, A., Sanyal, J. \& Chen, J. (2016). Characterizing provenance in visualization and data analysis: an organizational framework of provenance types and purposes. IEEE TVCG, 22(1), 31-40.

Robinson, John A. (1981). Personal narratives reconsidered. Journal of American Folklore, 94, 58-85. doi: 10.2307/540776

Segel, E. \& Heer, J. (2010). Narrative visualization: telling stories with data. IEEE TVCG, 16(6), 1139-1148.

Tufte, E.R. \& Graves-Morris, P.R. (1983). The visual display of quantitative information, 2(9). Cheshire, CT: Graphics press. 
Tulving, E. (1972). Episodic and semantic memory 1. Organization of Memory. London: Academic, 381(4), 382-404.

Worthham, Stanton. (2001). A dialogic approach to discourse. Narratives in Action: A Strategy for Research and Analysis. New York: Teachers College Press. 17-47.

Valle, M. (2015). Visualization and art. Retrieved from http:// mariovalle.name/visualization/VizArt.html

van Wijk, J.J. (2005). The value of visualization. Proceedings of IEEE Visualization. 79-86.

Viégas, F.B. \& Wattenberg, M. (2007). Artistic data visualization: Beyond visual analytics. Online Communities and Social Computing, 182-191. doi: 10.1007/978-3-540-73257-0_21

Ziemkiewicz, C. \& Kosara, R. (2008). The shaping of information by visual metaphors. IEEE TVCG, 14(6), 1269-1276.

\section{About the authors}

Alice Thudt is a PhD candidate in Computational Media Design, pursuing her research at the InnoVis Group at the University of Calgary. She is interested in using visualization to create personally meaningful artefacts from personal data.

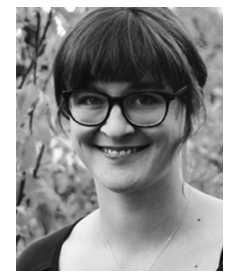

Recent projects have explored visualizations for autobiographical storytelling, the design of visualization techniques to reflect autobiographical memories, as well as the creation of visual mementos. She is further interested in democratizing the creation of visualization to a wider audience of non-experts.

Email: alice.thudt@googlemail.com

Charles Perin is a Lecturer in the department of Computer Science at City, University of London, United Kingdom. He is part of the giCentre and conducts research in information visualization and human computer interaction. He was previously a postdoctoral researcher at the University of Calgary, Canada. His research interests

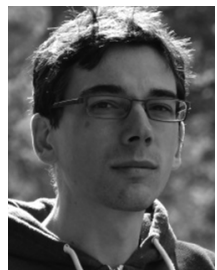
include the design and study of new interactions for information visualization, understanding how people use and interact with visualizations in their everyday lives, and human-centered design for specific applications such as sports and health. Perin has a PhD in computer science from Inria and CNRS-LIMSI at the University of Paris-Sud.

Email: charles.perin@city.ac.uk

Wesley Willett is an Assistant Professor of Computer Science at the University of Calgary. His interests span information visualization, social computing, new media, and human computer interaction, and his research focuses on pairing data and interactivity to support collaboration, learning, and discovery. At Calgary, Dr. Willett leads the Data Experience Lab, and is a faculty member in the department of Computer Science and the Computational Media Design program. His recent work has explored how communities can collaborate to collect and analyze data and how visualization can help people better understand personal data like digital notes.

Email:wj@wjwillett.net

Sheelagh Carpendale is a professor at the University of Calgary where she holds a Canada Research Chair in Information Visualization and the NSERC/AITF/SMART Industrial Research Chair in Interactive Technologies. She is the recipient of many awards including the NSERC Steacie and the British BAFTA. Her research draws upon her combined backgrounds in computer science and visual arts, benefiting from the interdisciplinary cross-fertilization to enable the design of innovative, people-centred information technologies. By studying how people interact with information both in work and social practices, she works towards designing more natural, accessible and understandable interactive visual representations of data. She combines information visualization, visual analytics and human-computer interaction with innovative interaction techniques to better support the everyday practices of people who are viewing, representing, and interacting with information.

Email: sheelagh@ucalgary.ca 


\section{Appendix}

Example personal visual narratives analyzed in this paper:

[E1] Clark, T. (2011) Atlas of the Habitual. Retrieved from: http://www.tlclark.com/ Atlas-of-the-Habitual

[E2] Felton, N. (2014) Feltron Annual Reports 2014. Retrieved from: http://feltron.com/

[E3] Heinicker, P. (2015) Good Night SMS. Retrieved from: http://paulheinicker.com/goodnightsms/

[E4] Lewis, K.H. (n.d.) 201 Days. Retrieved from: http:// katiehollandlewis.com/portfolio/201-days/

[E5] Kuo, A. (2010) Wheel of Worry. Retrieved from: http://www.nytimes.com/imagepages/2010/05/16/ magazine/16fob-idealab.html

[E6] Lupi, G. \& Posavec, S. (2015) Dear Data: Princeton Architectural Press, 244-245

[E7] McLean, K. (2010) Tactile Emotional Map. Retrieved from: http://sensorymaps.com/portfolio/ emotional-tactile-map/

[E8] McLean, K. (2012) Smell Map of Newport. Retrieved from: http://sensorymaps.com/portfolio/ newport/

[E9] Noveltysin. (2015) Heart Rate During Sex. Retrieved from: http://imgur.com/gallery/ GfRpoZb

[E10] Perin, C. (2015). A Personal Data Perspective on a CHI Deadline. Retrieved from: http://charles. perin.free.fr/misc/chi16deadline/
[E11] Sesikipai (2014). Heartrate during marriage proposal. Retrieved from: https://www.reddit. $\mathrm{com} / \mathrm{r} /$ dataisbeautiful/comments/201rfe/ heart_rate_bpm_during_marriage_proposal_oc/

[E12] Shelley, W. (2008) Autobiography, ver 2. Retrieved from: http://www.wardshelley.com/paintings/ pages/autobiography.html

[E13] Superremmjay. (2015). Weight loss over 20 months. Retrieved from: https://www.reddit. $\mathrm{com} / \mathrm{r} /$ dataisbeautiful/comments/3lxvxw/i_plotted_my_weight_loss_over_20_months_and/

[E14] Thudt, A. (2014) Shades of My Memories. Retrieved from: http://alicethudt.de/?port=shadesof-my-memories

[E15] Thudt, A. (2014) visits-Peru Trip 2013. Retrieved from: http://v.isits.in/

[E16] Vo, L. T. (2015) Rocky Beginnings. Retrieved from: http://quantifiedselfie.us/nyc365/

[E17] Vo, L. T. (2013) Quantified Breakup-Instagram Sapfest. Retrieved from: http://quantifiedbreakup. tumblr.com/

[E18] We, R. (n.d.) 8 Years of Dating Data. Retrieved from: http://www.robinwe.is/visualizations/dating. html

[E19] Wilson, J. L. (2010) The Grasses of America. Retrieved from: http://cargocollective.com/ julialineswilson/Weaving-the-Grasses-of-America

[E20] Woodruff, A. (2010) 2010 Paths Travelled. Retrieved from: https://www.flickr.com/ photos/awwoodruff/sets/72157625730585722/ with/5316713253/ 